This article was downloaded by: [EPFL Bibliothèque]

On: 07 May 2014, At: 07:02

Publisher: Taylor \& Francis

Informa Ltd Registered in England and Wales Registered Number: 1072954 Registered office: Mortimer House, 37-41 Mortimer Street, London W1T 3J H, UK

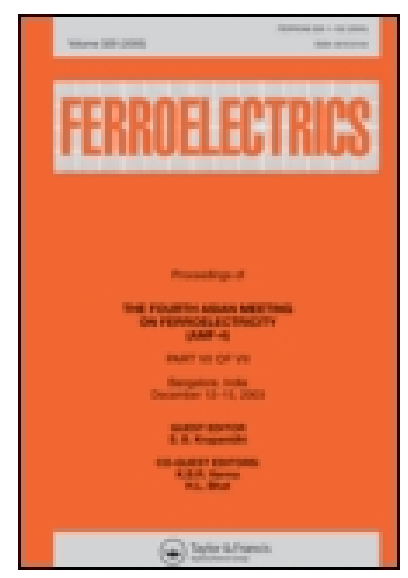

\title{
Ferroelectrics
}

Publication details, including instructions for authors and subscription information:

http:// www. tandfonline.com/loi/ gfer20

\section{Superdomain Structure in Epitaxial Tetragonal PZT Thin Films Under Tensile Strain}

\author{
L. Feigl $^{a}$, L. J. McGilly $\&$ N. Setter ${ }^{a}$ \\ ${ }^{a}$ Ceramics Laboratory, EPFL - Swiss Federal Institute of Technology, \\ Lausanne, $\mathrm{CH}$ 1015, Switzerland \\ Published online: 01 May 2014.
}

To cite this article: L. Feigl, L. J. McGilly \& N. Setter (2014) Superdomain Structure in Epitaxial Tetragonal PZT Thin Films Under Tensile Strain, Ferroelectrics, 465:1, 36-43, DOI: 10.1080/00150193.2014.893802

To link to this article: http:// dx.doi.org/ 10.1080/00150193.2014.893802

\section{PLEASE SCROLL DOWN FOR ARTICLE}

Taylor $\&$ Francis makes every effort to ensure the accuracy of all the information (the "Content") contained in the publications on our platform. However, Taylor \& Francis, our agents, and our licensors make no representations or warranties whatsoever as to the accuracy, completeness, or suitability for any purpose of the Content. Any opinions and views expressed in this publication are the opinions and views of the authors, and are not the views of or endorsed by Taylor \& Francis. The accuracy of the Content should not be relied upon and should be independently verified with primary sources of information. Taylor and Francis shall not be liable for any losses, actions, claims, proceedings, demands, costs, expenses, damages, and other liabilities whatsoever or howsoever caused arising directly or indirectly in connection with, in relation to or arising out of the use of the Content.

This article may be used for research, teaching, and private study purposes. Any substantial or systematic reproduction, redistribution, reselling, loan, sub-licensing, systematic supply, or distribution in any form to anyone is expressly forbidden. Terms \& Conditions of access and use can be found at http://www.tandfonline.com/page/termsand-conditions 


\title{
Superdomain Structure in Epitaxial Tetragonal PZT Thin Films Under Tensile Strain
}

\author{
L. FEIGL, ${ }^{*}$ L. J. McGILLY, AND N. SETTER \\ Ceramics Laboratory, EPFL - Swiss Federal Institute of Technology, Lausanne, \\ CH 1015, Switzerland
}

\begin{abstract}
The a/c domain pattern of tetragonal $\mathrm{PbZr}_{0.10} \mathrm{Ti}_{0.90} \mathrm{O}_{3}$ thin films under tensile misfit strain is investigated by piezoresponse force microscopy and X-ray diffraction. The results show a hierarchical ordering of the dense a/c domain structure into larger superstructures. The latter exhibit a preferred orientation and occasionally form distinct patterns such as flux closure loops of the net polarization. Additionally, the residual strain is measured with reciprocal space maps and the a-domain fraction is determined by theoretical calculations and correlated with results from rocking curve scans.
\end{abstract}

\section{Introduction}

Domain walls strongly change, or can even dominate, the properties of ferroelectric materials [1]. Since the discovery of interesting properties, such as domain wall conductivity in ferroelectric thin films [2, 3], they are even more the focus of interest, firstly due to the scientific endeavour to understand the underlying physical effects but also due to their potential application in novel types of electronic devices. In order to examine such effects, the domain structure has to be created in a controlled way. One possibility is to induce a specific amount of tensile misfit strain in thin films by depositing them on an accordingly suitable substrate material. This leads to the formation of ferroelastic domains, which can form only in two directions due to elastic confinements by the substrate. The amount of misfit strain determines the density of the domains.

In this report, $\mathrm{PbZr}_{0.10} \mathrm{Ti}_{0.90} \mathrm{O}_{3}$ (PZT) thin films are investigated, wherein the composition is close to the prototypic ferroelectric $\mathrm{PbTiO}_{3}$. Its strong tetragonality gives a wide misfit window to control its $a$-domain structure and should also promote a well-defined a-domain geometry.

\section{Experimental}

Heterostructures comprising a $30 \mathrm{~nm}$ thick $\mathrm{SrRuO}_{3}$ (SRO) bottom electrode layer and a $30 \mathrm{~nm}$ thick $\mathrm{PbZr}_{0.10} \mathrm{Ti}_{0.90} \mathrm{O}_{3}$ (PZT) ferroelectric layer were grown by pulsed laser deposition onto a $\mathrm{SmScO}_{3}$ (SSO) substrate (CrysTec GmbH, Germany) with a miscut angle of $0.1^{\circ}$ towards the [1 $1 \overline{0}$ ] direction. The pseudocubic lattice parameter of SRO is $0.3933 \mathrm{~nm}$

Received September 2, 2013; in final form September 23, 2013.

*Corresponding author. E-mail: ludwig.feigl@epfl.ch

Color versions of one or more of the figures in the article can be found online at www.tandfonline.com/gfer. 

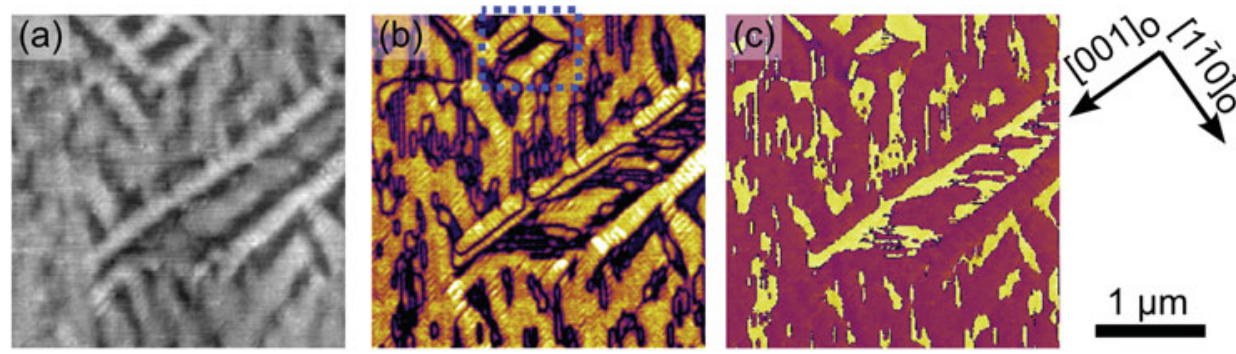

Figure 1. (a) Topography, (b) PFM amplitude and (c) PFM phase showing both the narrow a/c domain structure of the ferroelectric PZT thin film and the arrangement into superdomains. The z-range of the topography is $3 \mathrm{~nm}$.

[4], the tetragonal axes of PZT are $a=0.3914 \mathrm{~nm}$ and $c=0.4138 \mathrm{~nm}$ [5] and the lattice parameters of the perovskite unit cell of the orthorhombic SSO are $a=0.3988 \mathrm{~nm}$ and $b=0.3992 \mathrm{~nm}$ [6] (along the orthorhombic [110] and [001] direction, respectively). Prior to growth the SSO was annealed and etched in buffered $\mathrm{HF}$ and $\mathrm{NaOH}$ [7]. The SRO and PZT layers where grown at $T_{\mathrm{SRO}}=625^{\circ} \mathrm{C}$ and $T_{\mathrm{PZT}}=575^{\circ} \mathrm{C}$ at an oxygen pressure of $p_{\mathrm{O} 2, \mathrm{SRO}}=0.145 \mathrm{mbar}$ and $p_{\mathrm{O} 2, \mathrm{PZT}}=0.250 \mathrm{mbar}$ with the $\mathrm{KrF}$ excimer laser having a repetition rate of $2 \mathrm{~Hz}$ and $3 \mathrm{~Hz}$ respectively and an energy density of $1 \mathrm{~J} / \mathrm{cm}^{2}$. Piezoresponse force microscopy (PFM) was performed using an Asylum Research Cypher AFM, X-ray diffraction (XRD) was done with a Bruker D8 DISCOVER diffractometer.

\section{Results and Discussion}

Figure 1 shows a typical PFM image of the PZT films. Two different kinds of contrast are visible in the PFM amplitude: firstly, the partially curved dark lines are revealed by the PFM phase as a border where the out-of plane polarization changes orientation by $180^{\circ}$. Secondly, there is a weaker contrast within the bright areas, caused by a dense $a / c$ domain structure. This is in contrast to the growth of $\mathrm{PbTiO}_{3}$, which grows with a purely in-plane polarization on this kind of substrate due to its larger mismatch [8]. The a/c domain structure generates strain which leads to a deformation of the surface [9] as observed in the topography image. In spite of this deformation, the RMS roughness is only about $400 \mathrm{pm}$ on the $3 \times 3 \mu \mathrm{m}^{2}$ scan area, evidencing the excellent film quality.

The dense $\mathrm{a} / \mathrm{c}$ domain pattern has a periodicity of about $20-40 \mathrm{~nm}$ and is continuous over an area of width about 100-200 nm but with length up to 1-2 $\mu \mathrm{m}$. Within one area both the in-plane and the out-of-plane orientations of the polarization remain constant. Therefore an effective, large scale, or net, polarization can be attributed to such an area by simple addition of the local polar vectors. When passing from one area to the next, the direction of either the net out-of-plane or of the net in-plane polarization changes. Due to these properties, these areas could be considered as 'superdomains', giving rise to an ordering on two different length scales covered by the dimensions of both structures [10-12].

The orientation of the boundaries between the superdomains is not arbitrary. The dense $\mathrm{a} / \mathrm{c}$ domain structure follows the crystallographic axes of the substrate due to the misfit strain exerted along these directions. Therefore the boundary between them is determined by the $a_{1} / a_{2}$ domain wall resulting in an orientation along the two pseudocubic [110] $]_{\mathrm{pc}}$ directions [13]. 
For the boundaries given by the change of the out-of-plane polarization, four possible orientations exist. Two of them correspond to the ones given by the $a_{1} / a_{2}$ domain wall discussed above, which now additionally exhibits a change of the out-of-plane orientation. The second set of boundaries is exactly perpendicular to the narrow $\mathrm{a} / \mathrm{c}$ domain pattern.

However, it is evident that the two possible orientations are not uniformly distributed. Instead they show a preferred orientation along the [001] direction of the substrate. This orientation preference can be attributed to the anisotropy of the substrate lattice parameters. At high temperature, the lattice parameter of SSO along the [001] direction is slightly larger, which gives rise to a preferred nucleation of domains in this direction in order to release the strain. Therefore the $a / c$ domain pattern develops first along the [001] direction during cooling after growth. The $a$-domains that form along the [110] direction have to comply with the already existing pattern. Therefore their growth is impeded by the perpendicular structure thus preventing the extension of the $a / c$ domain structure along the [110] direction. These kinetics can explain the observed anisotropy, even though the mismatch difference between the film and the two substrate axes can be neglected at room temperature.

Since the superdomain boundaries should provide clues about the overall structure, it is worth looking closer into their fine-structure. An enlargement, of the central area from Fig. 1, is shown in Fig. 2, allowing closer analysis of the existing superdomain boundaries.

The boundary of type (1) is characterized by a change of the orientation of both the $c$-domain and $a$-domain polarizations as drawn schematically in Fig. 3(1). If the $c$-domain polarization changes its orientation, the $a$-domains must also change, either the orientation of their polarization, or the domain wall tilt direction e.g. a change of the domain wall from (101) to (101). In order to have a compatible domain wall tilt direction between the two different $a$-domains across the boundary, the polarization direction of the $a$-domains changes by $90^{\circ}$ as shown schematically in Fig. 3(1).

In the case of the boundaries as depicted in Fig. 2(2), the c-domain polarization remains the same, while only a change in the orientation of the a-domains is observed. Since the orientation of the in-plane polarization of the narrow a-domains cannot be resolved within the current PFM setup, two scenarios can be envisaged (Fig. 3(2a) and (2b) with the only difference being the reversal of the a-domain polarization on the left-hand side of the superdomain boundary which thus in turn changes the domain wall tilt direction. However, since the polarization of the c-domain doesn't change, this results in a non-compatible domain wall tilt across the superdomain boundary (Fig. 3(2b)). This mechanical incompatibility would cause a large elastic strain, making this kind of behaviour energetically costly. If the $a$-domain orientation is assumed as in Fig. 3(2a), this mechanical incompatibility is
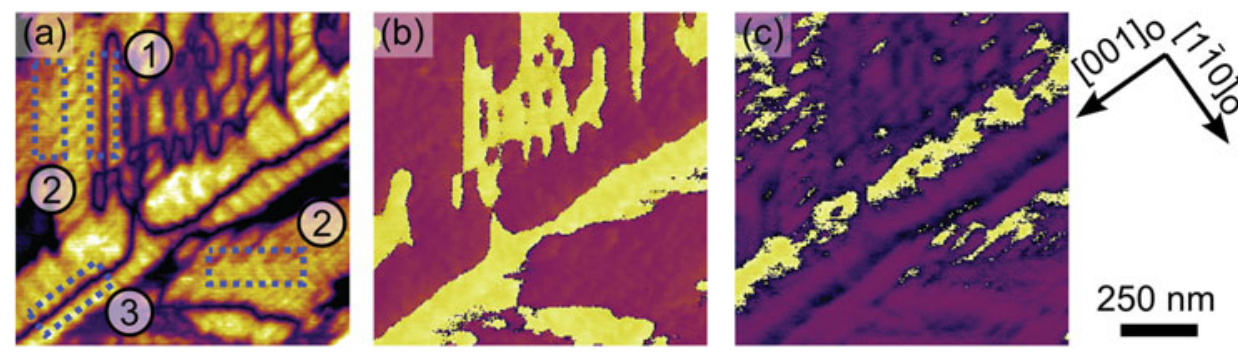

Figure 2. (a) Out-of-plane PFM amplitude and (b) phase and (c) in-plane PFM phase showing the complex a/c domain pattern in a close-up of the center in Fig. 1. The framed areas designate different kinds of boundaries, discussed in the text and drawn schematically in Fig. 3. 


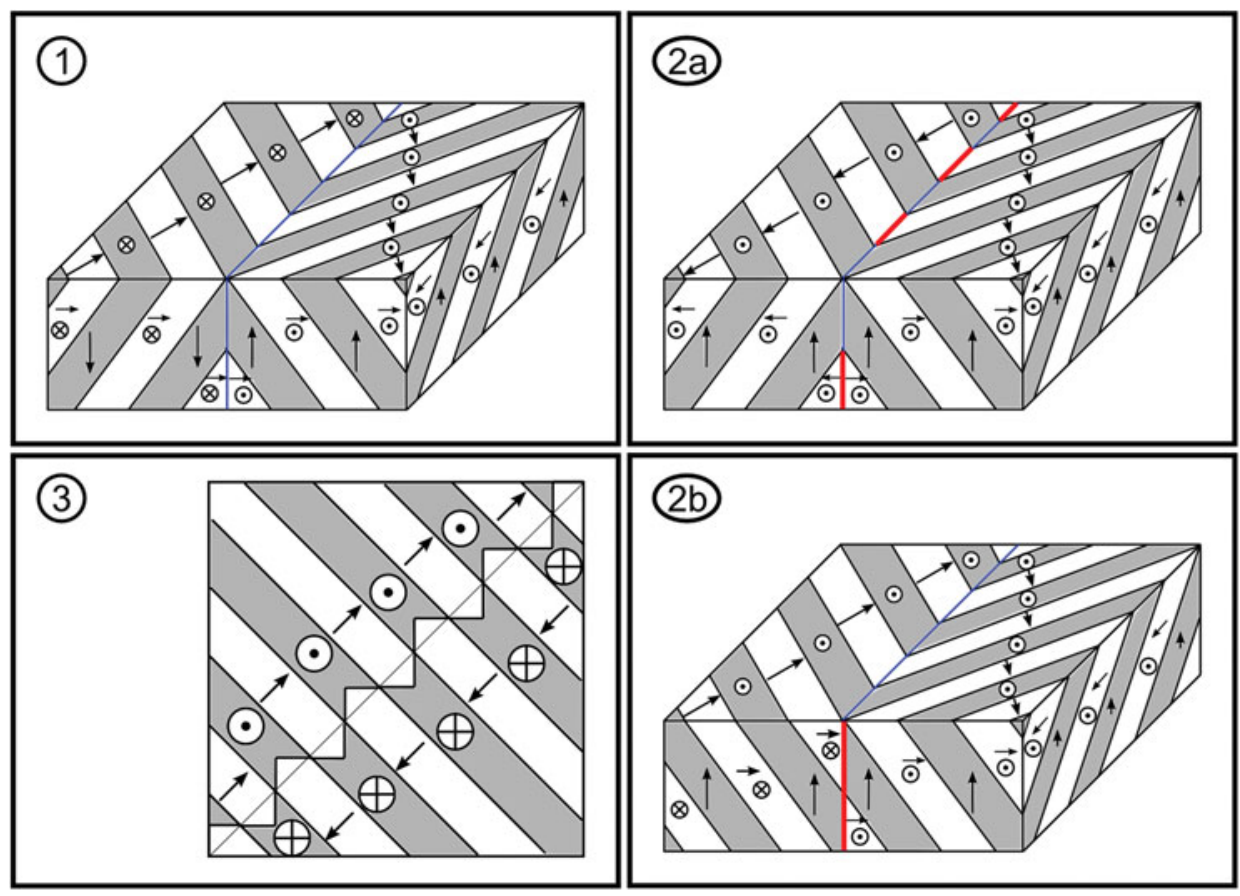

Figure 3. Schematic representations showing the different kinds of boundaries as observed in Figure 2. A blue boundary designates compatible conditions, whereas at the place of a thick red boundary either mechanical or electrostatic incompatibilities exist.

avoided. However, the price for this is that the polarization component perpendicular to the boundary is either head-to-head or tail-to-tail, creating a charged interface for the $a_{1} / a_{2}$ domain wall. Due to the aforementioned experimental indistinguishability of these two scenarios it is not possible to determine which one occurs. However, both scenarios will involve an incompatible component, which makes this type of boundary a likely candidate for unique properties related to domain walls. The possibility of partially charged domain walls in the second scenario makes the occurrence of high domain wall conductivity very likely [14].

Boundary (3) exhibits a jagged shape and is turned by $45^{\circ}$ with respect to the other boundaries. Across this boundary, both the in-plane and the out-of-plane polarization components change direction. Additionally, this boundary is accompanied by a strong deformation of the surface (see Fig. 1a). This strong surface deformation indicates an elastic incompatibility of the two domain patterns. A state where $a$-domains terminate in a needle tip would be able to create a strain which could result in such a surface deformation [15]. Therefore a domain pattern as imagined in Fig. 3(3) has to be assumed wherein, across the superdomain boundary, polar orientations of both a- and c-domains change i.e. a change in net polarization. However now the two sub-patterns are offset with respect to one another i.e. the $a$-domains meet the $c$-domains across the superdomain boundary. This 'phase' offset in turn causes the observed surface deformation. A possible explanation for this observed behaviour could lie in the fact that the two domain patterns, most likely, nucleate at different points of the sample. When they grow and meet each other, the mutual clamping within each pattern makes it energetically elaborate to shift the phase of the whole pattern, 

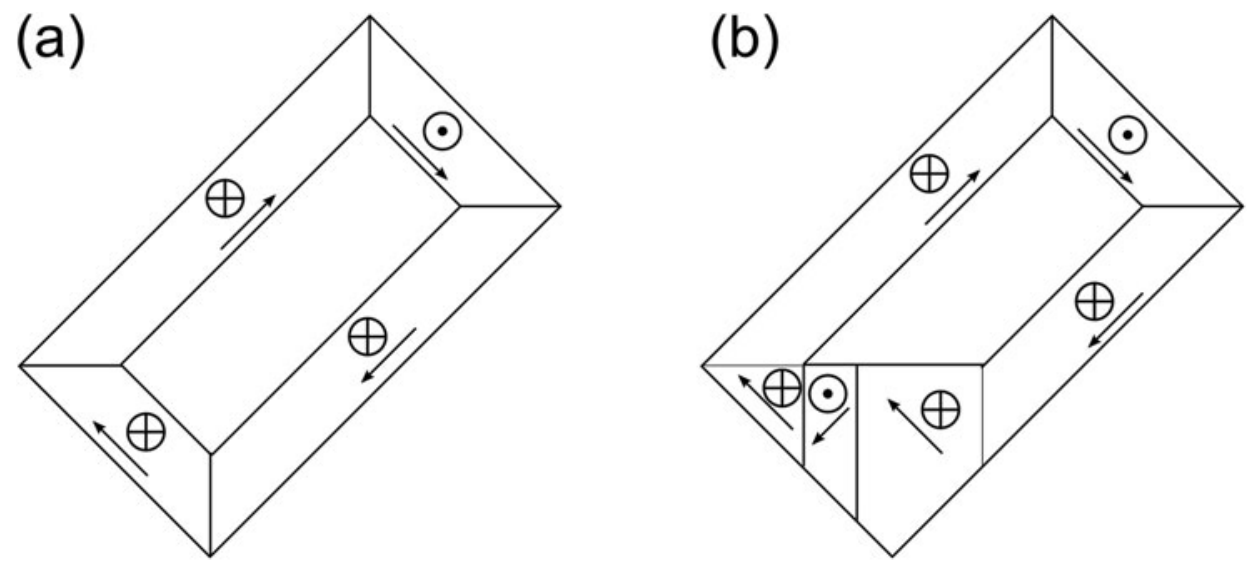

Figure 4. Schematic of (a) an ideal flux closure loop comprised of different superdomains and (b) schematic drawing of the actual structure as framed in the top portion of Fig. 1

therefore it seems to be favourable for such structures to introduce the surface deformation instead. Without this domain pattern phase shift, the superdomain boundary would simply represent a $180^{\circ}$ domain wall which would not cause any surface deformation. All the other boundaries, though many structures may occur, consist of combinations of the discussed boundaries.

One distinct feature of the combination of superdomains is a flux closure structure $[16,17]$ existing over a large scale as observed in the top portion of Fig. 1b. The flux closure does not exist around a single point in order to avoid the associated singularity [18]. This structure is drawn schematically in Fig. 4a. However, the observed structure is not perfect, since other boundaries occur as well, giving rise to a similar, but more complex structure which still manages to maintain flux closure as is seen in Fig. 4b.

The formation of the domain structure releases the misfit strain imposed by the substrate. The amount of released strain depends on the specific domain pattern dimensionality, that is, whether the periodic nature of the $a / c$ domain pattern is 2 dimensional (2D) or extends in all three spatial directions (3D). For thin films, it is usually assumed, that locally only one type of $a$-domain exists i.e. the polarization exists only in 2 dimensions and not the third, whereas for thick films a $3 \mathrm{D}$ variant is also possible. The fraction of $a$-domains is different for both kind of structures and can be calculated for a tetragonal film by means of the Landau theory from the relative coherency strain [19]:

$$
\Phi=\frac{a_{\text {film }}-a_{\text {substrate }}}{a_{\text {film }}-c_{\text {film }}}
$$

In the case of the 2D variant the fraction of $a$-domains is:

$$
\alpha_{2 \mathrm{D}}=(1+v) \Phi
$$

with $v$ being the Poisson ratio. For a 3D variant the fraction of $a$-domains would be:

$$
\alpha_{3 \mathrm{D}}=2 \Phi .
$$




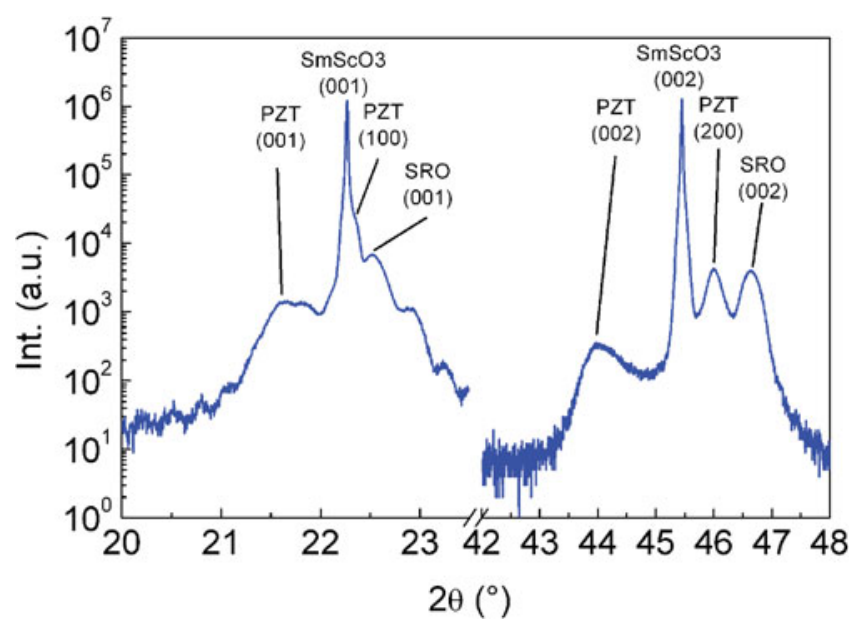

Figure 5. $\theta-2 \theta$ XRD scan of the epitaxial heterostructure, showing the first and second order peaks. The peaks are well separated in the second order, which allows addressing them individually in rocking curve scans. Thickness fringes around the first order peaks indicate a very good interface quality.

As it can be seen from (2) and (3), the two fractions should be quite different. When calculating the expected fractions in PZT10/90 on SSO by using the corresponding material parameters, one would expect $\alpha=44 \%$ for the $2 \mathrm{D}$ variant and $\alpha=68 \%$ for the 3D variant.

Since it is not possible to resolve the width of the $a$-domains with PFM, XRD was employed to estimate the fraction of a-domains. Fig. 5 shows the $\theta-2 \theta$ scans of the first and second order diffraction peaks. For the second order, the substrate, SRO electrode and PZT $c$-domain as well as the $a$-domain peaks are well separated. This easily allows measurement of the rocking curves of the respective peaks. From such rocking curves the fraction of $a$-domains is determined to be approximately $69 \%$, which is nearly a perfect match to the value calculated for the $3 \mathrm{D}$ pattern. This close agreement could suggest that $a$-domain crossings occur in the film, signifying a 3D domain variant.

In order to calculate the strain state of the films both the in-plane and out-of-plane lattice parameters were determined from reciprocal space maps (Fig. 6). It can be seen that the SRO is perfectly strained to the substrate, which is important to propagate the epitaxial strain from the substrate to the PZT layer. Even though the in-plane $a$-axes of the PZT layer are relaxed due to the formation of the observed dense $a / c$ domain pattern, the lattice parameter is still partially strained to a value of $0.3946 \mathrm{~nm}$ which corresponds to a residual strain of about $0.8 \%$. This residual strain is a measure of the energy needed for the creation of an additional a-domain. Since the tetragonality of the chosen PZT composition is quite high, the deformation around an $a / c$ domain wall is large. This causes a high energetic cost for the creation of an additional $a$-domain, which is energetically unfavourable compared to the observed residual strain.

\section{Summary}

PZT films were grown on SSO substrates. This substrate imposes tensile strain which creates a dense $a / c$ domain pattern within the PZT film. The fraction of $a$-domains as measured by XRD rocking curves suggests the formation of a 3D variant domain structure. On 


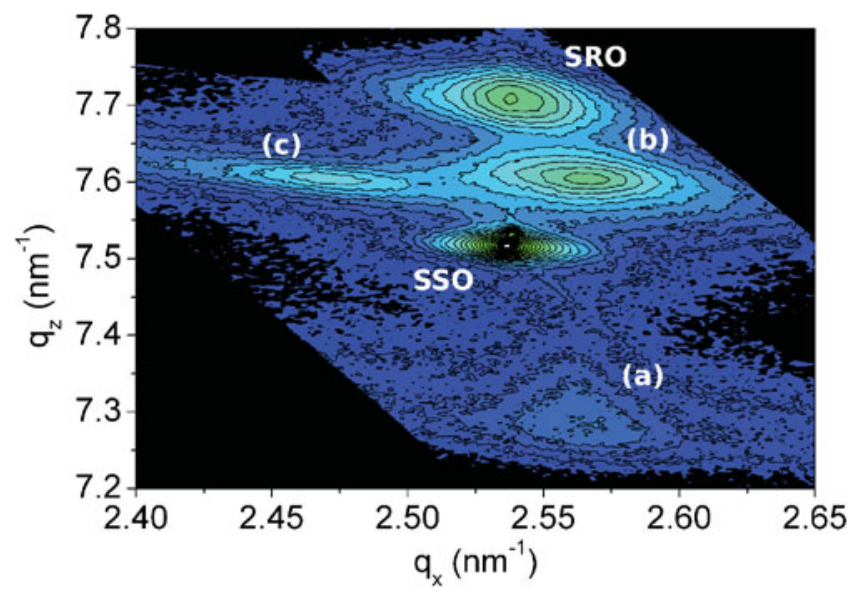

Figure 6. XRD reciprocal space map of the SSO substrate, the SRO films and the different peaks originating from the PZT layer: (a) c-domain, (b) $a_{1} / a_{2}$ axes of the a-domain, and (c) a/c axes of the a-domain. The SRO is grown coherently, whereas the PZT shows partial relaxation.

a larger scale, the a/c domain structure arranges into well-defined areas with an effective net polarization direction, which can thus be considered as superdomains. This hierarchical structure gives rise to an ordering on two size scales, effectively reducing the misfit strain throughout the film. The boundaries between the superdomains bear mechanical and electrostatic incompatibilities, making these regions promising to exhibit new functional properties. One type of boundary presumably exhibits partially charged domain walls where electrical conduction could potentially be enhanced compared to uncharged domain walls and the bulk. Additionally, the superdomains can arrange into large-scale structures, one observed example being a combination which results in a flux closure loop of the in-plane polarization component.

\section{Funding}

This research has received funding from the European Research Council under the EU 7th Framework Programme (FP7/2007-2013) / ERC grant agreement $n^{\circ}$ [268058] and from the Swiss State Secretariat for Education, Research and Innovation (support for the COSTEuropean Cooperation in the Field of Scientific and Technical Research; 0904 Action).

\section{References}

1. S. Wada, K. Yako, H. Kakemoto, T. Tsurumi, and T. Kiguchi, Enhanced piezoelectric properties of barium titanate single crystals with different engineered-domain sizes. $J$ Appl Phys., 98, 014109-1-7 (2005).

2. J. Seidel, L. W. Martin, Q. He, Q. Zhan, Y-H. Chu, A. Rother, M. E. Hawkridge, P. Maksymovych, P. Yu, M. Gajek, N. Balke, S. V. Kalinin, S. Gemming, F. Wang, G. Catalan, J. F. Scott, N. A. Spaldin, J. Orenstein, and R. Ramesh, Conduction at domain walls in oxide multiferroics. Nat Mater., 8, 229-234 (2009).

3. J. Guyonnet, I. Gaponenko, S. Gariglio, and P. Paruch, Conduction at Domain Walls in Insulating $\mathrm{Pb}\left(\mathrm{Zr}_{0.2} \mathrm{Ti}_{0.8}\right) \mathrm{O}_{3}$ Thin Films. Adv Mater., 23, 5377-5382 (2011). 
4. S. Yamanaka, T. Maekawa, H. Muta, T. Matsuda, S.-I. Kobayashi, and K. Kurosaki, Thermophysical properties of $\mathrm{SrHfO}_{3}$ and $\mathrm{SrRuO}_{3}$. J Solid State Chem., 177, 3484-3489 (2004).

5. Landoldt-Börnstein, Numerical Data and Functional Relationships in Science and Technology, New Series vol. III/16a. New York: Springer, (1981).

6. B. Velickov, V. Kahlenberg, R. Bertram, and M. Bernhagen, Crystal chemistry of $\mathrm{GdScO}_{3}$, $\mathrm{DyScO}_{3}, \mathrm{SmScO}_{3}$ and $\mathrm{NdScO}_{3}$. Z Kristallogr., 222, 466-473 (2007).

7. J. E. Kleibeuker, G. Koster, W. Siemons, D. Dubbink, B. Kuiper, J. L. Blok, C-H. Yang, J. Ravichandran, R. Ramesh, J. H. tenElshof, D. H. A. Blank, and G. Rijnders, Atomically Defined Rare-Earth Scandate Crystal Surfaces. Adv Funct Mater., 20, 3490-3496 (2010).

8. F. Borodovka, I. Gregora, A. Bartasyte, S. Margueron, V. Plausinaitiene, A. Abrutis, and J. Hlinka, Ferroelectric nanodomains in epitaxial $\mathrm{PbTiO}_{3}$ films grown on $\mathrm{SmScO}_{3}$ and $\mathrm{TbScO}_{3}$ substrates. J Appl Phys., 113, 187216-1-7 (2013).

9. J. S. Speck, A. C. Daykin, A. Seifert, A. E. Romanov, and W. Pompe, Domain configurations due to multiple misfit relaxation mechanisms in epitaxial ferroelectric thin films. III. Interfacial defects and domain misorientations. J Appl Phys., 78, 1696-1706 (1995).

10. G. Arlt and P. Sasko, Domain configuration and equilibrium size of domains in $\mathrm{BaTiO}_{3}$ ceramics. J Appl Phys., 51, 4956-4960 (1980).

11. F. Bai, J. Li, and D. Viehland, Domain engineered states over various length scales in (001)oriented $\mathrm{Pb}\left(\mathrm{Mg}_{1 / 3} \mathrm{Nb}_{2 / 3}\right) \mathrm{O}_{3}-\mathrm{x} \% \mathrm{PbTiO}_{3}$ crystals: Electrical history dependence of hierarchal domains. J Appl Phys., 97, 054103-1-7 (2005).

12. L. J. McGilly and J. M. Gregg, Scaling of superdomains bands in ferroelectric dots. Appl Phys Lett., 98, 132902-1-3 (2011).

13. W. J. Merz, Domain Formation and Domain Wall Motions in Ferroelectric $\mathrm{BaTiO}_{3}$ Single Crystals. Phys Rev., 95, 690-698 (1954).

14. T. Sluka, A. K. Tagantsev, P. Bednyakov, and N. Setter, Free-electron gas at charged domain walls in insulating $\mathrm{BaTiO}_{3}$. Nat Commun., 4, 1808 (2013).

15. W. Schranz and I. Rychetsky, Domains and domain wall interactions near the improper ferroelastic phase transition of KSCN. J Phys: Condens Mater., 5, 3871-3882 (1993).

16. R. G. P. McQuaid, L. J. McGilly, P. Sharma, A. Gruverman, and J. M. Gregg, Mesoscale fluxclosure domain formation in single-crystal $\mathrm{BaTiO}_{3}$. Nat Commun., 2, 404 (2011).

17. L-W. Chang, V. Nagarajan, J. F. Scott, and J. M. Gregg, Self-Similar Nested Flux Closure Structures in a Tetragonal Ferroelectric. Nano Lett., 13, 2553-2557 (2013).

18. L. J. McGilly, A. Schilling, and J. M. Gregg, Domain Bundle Boundaries in Single Crystal $\mathrm{BaTiO}_{3}$ Lamellae: Searching for Naturally Forming Dipole Flux-Closure/Quadrupole Chains. Nano Lett., 10, 4200-4205 (2010).

19. A. K. Tagantsev, L. A. Cross, and J. Fousek, Domains in Ferroic Crystals and Thin Films. New York Dordrecht Heidelberg London: Springer, (2010). 